\title{
MULTI-STEP OPTIMIZATION OF PARAMETERS IN THE XINANJIANG MODEL TAKING INTO ACCOUNT THEIR TIME SCALE DEPENDENCY
}

\author{
Xiao $\mathrm{LI}^{1}$ and Minjiao LU 2,3 \\ ${ }^{1} \mathrm{Ph}$. D. student, Dept. of Civil and Environmental Engineering, Nagaoka University of Technology (Nagaoka, \\ Niigata 940-2188, Japan) \\ ${ }^{2}$ Member of JSCE, Professor, Dept. of Civil and Environmental Engineering, Nagaoka University of Technology \\ (Nagaoka, Niigata 940-2188, Japan) \\ ${ }^{3}$ Chongqing Jiaotong University, Chongqing, China
}

\begin{abstract}
The objective of this study is to find an efficient way to optimize parameters in the Xinanjiang model automatically. According to the time scale dependent sensitivities, a multi-step optimization scheme is developed with the help of the SCEM-UA algorithm. Some case studies were designed to test the efficiency of the scheme. The tests show this scheme under ideal condition could converge to the true values of parameters to be optimized. For the actual practice, the multi-step optimization scheme is capable of searching the optimum parameter set for the Xinanjiang model, and the simulation results are acceptable.
\end{abstract}

Key Words : Multi-step optimization scheme, Time scale dependency, Xinanjiang model, Parameter calibration

\section{INTRODUCTION}

The Xinanjiang model developed by Zhao et $a l .{ }^{1)}$ has been widely applied in humid and semi-humid regions of China and many countries in the field of runoff simulation, hydrological prediction, water resources assessment, design flood estimation, water project programming, water quality accounting and so on. Usually the parameters are calibrated manually by a trial-and-error process. However, manual calibration requires the modelers having detailed understanding of the model structure and plenty of calibration experience. An expert modeler could obtain very good calibration results in a short time, while, for the inexperienced or untrained people, the manual calibration process may become time consuming and frustrating.

The development of computer technology makes it possible to determine parameter values of a model automatically without subjective judgment of modelers $^{2}$. For example, Rosenbrock ${ }^{3)}$ developed a local search method for finding the greatest or least value of a function of several variables are restricted to a given region, and compared the method with possible alternatives; Duan et $a l^{4}{ }^{4}$ developed a global optimization called the shuffled complex evolution (SCE-UA) method to deal with the peculiarities encountered in calibration of conceptual watershed models; based on the success of SCE-UA single-objective global optimization algorithm, Yapo et al. ${ }^{5)}$ presented an effective and efficient methodology, the MOCOM-UA algorithm, for solving the multiple-objective global optimization problem; Beven and Binley ${ }^{6}$ and Beven $^{7)}$ pointed out the importance of realistic assessment of uncertainty in hydrological prophecy for model calibration and validation, and described a methodology for calibration and uncertainty estimation of distributed models based on generalized likelihood measures, and so on.

Some of these methods had been applied for parameter calibration of Xinanjiang model. Chen et $a l^{8)}$ discussed the application of the multi-objective complex evolution (MOCOM-UA) algorithm coupled with the simplex method as an optimal solution for rainfall-runoff simulation of the Xinanjiang model in the upper reaches of the 
Hanjiang River Basin, and calculated the Pareto space of parameters and the prediction extent of the model. Hapuarachchi et al. ${ }^{9)}$ used the SCE-UA method for calibrating Xinanjiang watershed model using hydrological data of three catchments of different size and climatic conditions. Zhang et al. ${ }^{10)}$ selected three optimization algorithms: Rosenbrock method, Simplex method and Genetic algorithm, and compared their converge speed, the initial value and different composition in four basins. The past researches provided some useful advices and experiences for parameter optimization of the Xinanjiang model. However, there are still some problems needing to be considered. Hydrological model is a very complex system, there will be multiple local optimum values in the response surface of parameter space and the optimization process is easy to fall into one of local optimum area and can not search the global optimum values. Moreover, the possibilities for parameter interaction may make optimization process more difficult and time consuming.

In this study, the sensitivity of parameters of Xinanjiang model at different time scale is taken into account. The parameters were divided into three groups and then optimized group by group with the help of SCEM-UA algorithm. In this way, it could reduce the dimension of parameter space, and make the optimization process more effective and efficient.

\section{SCHEMATIC OF THE XINANJIANG MODEL}

The main feature of the Xinanjiang model is the concept of runoff formation on repletion of storage, which means that runoff is not produced until the soil moisture content of the aeration zone reaches the field capacity, and thereafter runoff equals the rain fall excess without further loss. Runoff so generated was separated into three components: surface runoff, interflow and groundwater. At last, the local runoff was transferred to the outflow in the outlet of basin. The simulation of outflow is consisted of four major parts:

(1) the evapotranspiration part which generates the deficit of the soil storage in three layers;

(2) the runoff production part which produces the runoff according to the rainfall and soil storage deficit which is divided into upper, lower and deeper layers;

(3) the runoff separation part which divides the determined runoff into three components: surface, subsurface and groundwater;

(4) the flow routing part which transfers the local
Table 1 Parameters and their physical meanings in the Xinanjiang model.

\begin{tabular}{|c|c|}
\hline Parameter & Physical meaning \\
\hline $\mathrm{Cp}$ & $\begin{array}{l}\text { Ratio of measured precipitation to actual } \\
\text { precipitation }\end{array}$ \\
\hline Cep & $\begin{array}{l}\text { Ratio of potential evapotranspiration to pan } \\
\text { evaporation }\end{array}$ \\
\hline $\mathrm{b}$ & $\begin{array}{l}\text { Exponent of the tension water capacity } \\
\text { curve }\end{array}$ \\
\hline imp & $\begin{array}{l}\text { Ratio of the impervious to the total area of } \\
\text { the basin }\end{array}$ \\
\hline WM & Areal mean tension water capacity $[\mathrm{mm}]$ \\
\hline WUM & Water capacity in the upper soil layer [mm] \\
\hline WLM & Water capacity in the lower soil layer [mm] \\
\hline $\mathrm{C}$ & Coefficient of deep evapotranspiration \\
\hline SM & $\begin{array}{l}\text { Areal mean of the free water capacity of } \\
\text { the surface soil layer }[\mathrm{mm}]\end{array}$ \\
\hline EX & Exponent of the free water capacity curve \\
\hline KI & $\begin{array}{l}\text { Outflow coefficients of the free water } \\
\text { storage to interfolw }\end{array}$ \\
\hline $\mathrm{KG}$ & $\begin{array}{l}\text { Outflow coefficients of the free water } \\
\text { storage to groundwater }\end{array}$ \\
\hline cs & Recession constant for channel routing \\
\hline ci & $\begin{array}{l}\text { Recession constant of the lower interflow } \\
\text { storage }\end{array}$ \\
\hline $\operatorname{cg}$ & $\begin{array}{l}\text { Daily recession constant of groundwater } \\
\text { storage }\end{array}$ \\
\hline
\end{tabular}

runoff components to the outflow in the outlet.

For the details of the Xinanjiang model, the reader is recommended to refer to Zhao et al. ${ }^{1)}$.

The inputs to Xinanjiang model are areal mean rainfall and measured pan evaporation, while the outputs are the discharge from the whole basin and the actual evapotranspiration from three soil layers. In the original Xinanjiang model, a catchment is usually divided into some sub-basins by Thiessen Polygon method. The runoff from each sub-basin is calculated from corresponding rainfall data independently and routed to the basin outlet by Muskingum method. The adjustment for rainfall data is not considered. However, in this study the model is used in a pure lumped way. The areal average rainfall is inputted to the model representing the whole catchment. In order to take into account the possible errors caused by gauge losses, altitudinal distribution of rainfall and the representativeness of the gauge network, an adjustment coefficient $(\mathrm{Cp})$ is introduced to derive adjusted rainfall from areal average rainfall. So there are 15 parameters in the modified Xinanjiang model in this study. Table 1 shows the detail of each parameter. 


\section{MUTI-STEP OPTIMIZATION SCHEME}

\section{(1) Sensitivity analysis}

The difficulties in parameter optimization of the Xinanjiang model mainly focus on two factors: (1)The number of parameters in Xinanjiang model needing to be calibrated is large, so that reducing the effective of optimization method. (2) The sensitivity of each parameter and interaction between parameters are complex, so that making the automatic calibration very difficult. For these, the parametric sensitivity is a vital issue needing to be investigated firstly.

The sensitivity analysis by $\mathrm{Lu}$ and $\mathrm{Li}^{11)}$ showed that the sensitivity of parameters in Xinanjiang model is influenced by the time scale. At annual scale, the parameters controlling data adjustment are most sensitive. After excluding these factors, parameters controlling runoff generation become sensitive together with several parameters controlling runoff routing at monthly and daily scale. And the parameters relating to runoff generation are less sensitive at all three time scales.

\section{(2) Optimization procedures}

Based on the information from sensitivity analysis, the total 15 parameters could be divided into three groups:

- Group 1: parameters for data adjustment, Cp and Cep, which are sensitive at annual scale.

- Group 2: parameters controlling runoff component separation and routing, SM, EX, KI, KG, cs, ci and $\mathrm{cg}$, which are sensitive at daily scale.

- Group 3: parameters controlling runoff generation, imp, b, WM, WUM, WLM and C, which are sensitive at annual scale when Cp and Cep are set to be constants.

A multi-step optimization scheme is given as follows and is illustrated in Fig. 1:

(1) Assume a set of commonly used parameters in group 2 and 3, optimize parameters of group 1 at annual scale using SCEM-UA algorithm.

(2) Set parameter of group 1 to be the optimum values got from step 1 while keep the parameters in group 3 the same. Then optimize the parameters of group 2 by the SCEM-UA algorithm at daily scale.

(3) The parameter values of group 1 and group 2 are set to be optimum values, and optimize the parameters of group 3 using the SCEM-UA algorithm at annual scale.

\section{(3) Transformation functions}

The multi-step optimization scheme is set to randomly generate sample points from a unit hypercube. Then using a series of functions, these

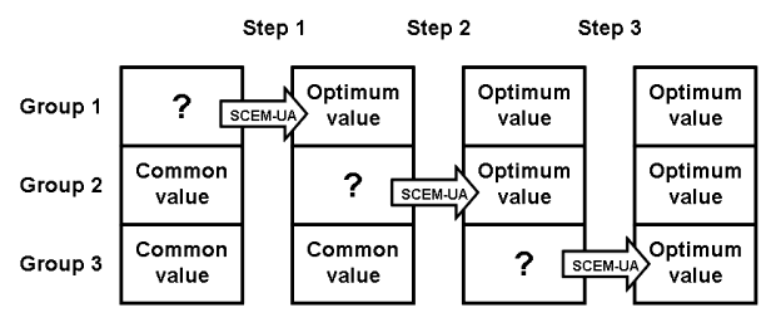

Fig.1 Flowchart of the multi-step optimization scheme.

Table 2 Transformation functions of parameters in the Xinanjiang model.

\begin{tabular}{|c|c|c|c|}
\hline \multirow{2}{*}{ Para. } & \multicolumn{2}{|c|}{ Range } & \multirow{2}{*}{ Transformation Function } \\
\hline & Min. & Max. & \\
\hline $\mathrm{Cp}$ & 0.8 & 1.2 & $\mathrm{Cp}=0.8+0.4 \mathrm{~A}_{1}$ \\
\hline Cep & 0.0 & 2.0 & $\mathrm{Cep}=2 \mathrm{~A}_{2}$ \\
\hline $\mathrm{b}$ & 0.1 & 0.3 & $\mathrm{~b}=0.1+0.2 \mathrm{~A}_{3}$ \\
\hline imp & 0.0 & 0.05 & $\mathrm{imp}=0.05 \mathrm{~A}_{4}$ \\
\hline WM & 110 & 200 & $\mathrm{WM}=110+90 \mathrm{~A}_{5}$ \\
\hline WUM & 10 & 30 & $\mathrm{WUM}=10+20 \mathrm{~A}_{6}$ \\
\hline WLM & 50 & 90 & $\mathrm{WLM}=50+40 \mathrm{~A}_{7}$ \\
\hline $\mathrm{C}$ & 0.1 & 0.3 & $\mathrm{C}=0.1+0.2 \mathrm{~A}_{8}$ \\
\hline SM & 1 & 30 & $\mathrm{SM}=1.0+29.0 \mathrm{~A}_{9}$ \\
\hline EX & 0.5 & 2.5 & $\mathrm{EX}=0.5+2.0 \mathrm{~A}_{10}$ \\
\hline KI & 0.001 & 0.95 & $\mathrm{KI}=0.001+0.95 \mathrm{~A}_{11}$ \\
\hline KG & 0.001 & $1-\mathrm{KI}$ & $\mathrm{KG}=0.001+0.95(1-\mathrm{KI}) \mathrm{A}_{12}$ \\
\hline cs & 0.01 & ci & $\mathrm{cs}=0.01+0.99 \mathrm{ciA}_{13}$ \\
\hline ci & 0.01 & $\mathrm{cg}$ & $\mathrm{ci}=0.01+0.99 \mathrm{cgA} \mathrm{A}_{14}$ \\
\hline $\mathrm{cg}$ & 0.97 & 0.995 & $\mathrm{cg}=0.97+0.025 \mathrm{~A}_{15}$ \\
\hline
\end{tabular}

sample points are transferred to corresponding parameter values of Xinanjiang model. The transformation functions for parameters to be optimized are listed in Table 2. The ranges of parameters are set to be wider than normal values in order to ensure that the global optimum values are contained in the parameter space and have chance to be searched.

\section{(4) Application of the SCEM-UA algorithm}

The key method in the multi-step optimization scheme is the SCEM-UA (Shuffled Complex Evolution Metropolis) algorithm which is a global optimization method developed by Duan et al. ${ }^{4}$. The SCEM-UA algorithm contains two algorithm parameters that need to be specified by the user. There are: the number of complexes/sequences, q, and the population size, s. Vrugt et al. 12),13) suggested that for simple problems with an uncorrelated or correlated Gaussian target distribution, relatively small values $(\mathrm{s} \leq 100$ and $\mathrm{q} \leq 5)$ will usually suffice, while in the case of complex-shaped posterior probability density distributions, the use of larger values $(\mathrm{s} \geq 250$ and 
$\mathrm{q} \geq 10$ ) will be appropriate. In this study, the parameters were set to be $q=15$ and $s=(2 n+1) q$, where $\mathrm{n}$ is the number of parameters to be optimized. And the Gelman and Rubin scale reduction factor of less than 1.2 indicates approximate convergence (For the details of this factor, see Vrugt et al. ${ }^{12)}$ ). The optimization process will stop when convergence criteria are satisfied. Otherwise, the process will stop until the maximum number of iterations is exceeded.

\section{(5) Statistical index}

The efficiency of Nash and Sutcliffe ${ }^{14)}$ is used to assess the precision of simulation result of Xinanjiang model using optimum values of parameters.

$$
N A S H=1-\frac{\sum_{i=1}^{n}\left(Q_{o}-Q_{s}\right)^{2}}{\sum_{i=1}^{n}\left(Q_{o}-Q_{\text {avg }}\right)^{2}}
$$

where $Q_{s}$ is the simulated runoff; $Q_{o}$ is the observed runoff; and $Q_{a v g}$ is the mean value of observed runoff.

For all of three optimization steps, the model runs at daily scale, while the objective functions are calculated using cumulative daily runoff at their relevant time scales. In order to avoid the effect from initial conditions, the data in the first year are not considered in the calculation of the modeling efficiency of Nash and Sutcliffe.

\section{CASE STUDIES}

\section{(1) Ideal condition}

It is clear that even though the measurement instruments are very sensitive and precision, the observed error could not be avoided completely. In order to reduce the impact of measurement errors, and to test the efficiency of the multi-step optimization scheme, a series of 'perfect' runoff data was used instead of the observed runoff as reference data. These perfect data were generated by Xinanjiang model directly with a set of common parameter values and the initial condition for this generation process was set to be saturated. The 7-year (1982-1988) daily observed precipitation data in Misai Basin $\left(118.0^{\circ}-119.0^{\circ} \mathrm{E}, 29.10^{\circ}-30.0^{\circ} \mathrm{N}\right)$, which located in the southeast of China with drainage area of $797 \mathrm{~km}^{2}$, were chosen to generate the perfect data (Fig.2).

The optimization process was carried out as describing above. Table 3 shows the result of each step and the final optimum set. Fig. 3 shows the fit


Fig.2 DEM and stations of the Misai Basin.

Table 3 Optimum values of parameters in the Xinanjiang model for perfect data.

\begin{tabular}{cccccc}
\hline \multirow{2}{*}{ Para. } & \multicolumn{5}{c}{ Parameter values } \\
\cline { 2 - 6 } Group 1: & True & Start from & Step 1 & Step 2 & Step 3 \\
Cp & 0.95 & & 0.9623 & 0.9623 & 0.9623 \\
Cep & 0.85 & & 0.9031 & 0.9031 & 0.9031 \\
Group 2: & & & & & \\
SM & 10 & 15.0 & 15.0 & 8.93 & 8.93 \\
EX & 1.2 & 1.5 & 1.5 & 1.1570 & 1.1570 \\
KI & 0.3 & 0.3 & 0.3 & 0.3388 & 0.3388 \\
KG & 0.4 & 0.4 & 0.4 & 0.4334 & 0.4334 \\
cs & 0.4 & 0.35 & 0.35 & 0.4014 & 0.4014 \\
ci & 0.8 & 0.85 & 0.85 & 0.7854 & 0.7854 \\
cg & 0.98 & 0.9 & 0.9 & 0.9806 & 0.9806 \\
Group 3: & & & & & \\
b & 0.2 & 0.2 & 0.2 & 0.2 & 0.2495 \\
imp & 0.01 & 0.01 & 0.01 & 0.01 & 0.0203 \\
WM & 120 & 160.0 & 160.0 & 160.0 & 117.41 \\
WUM & 20 & 20.0 & 20.0 & 20.0 & 15.32 \\
WLM & 60 & 60.0 & 60.0 & 60.0 & 75.17 \\
C & 0.1 & 0.1 & 0.1 & 0.1 & 0.0758 \\
Annual & & & 0.9996 & 0.9998 & 0.9999 \\
NASH & & & & & \\
Daily & & & 0.9906 & 0.9994 & 0.9993 \\
NASH & & & & &
\end{tabular}

of calculated daily runoff using optimum values of parameters and the perfect runoff. For convenience, only selected period (one year) of the hydrograph is shown here.

Compared with optimizing all 15 parameters directly without dividing into groups, the efficiency of multi-step optimization scheme is higher. Table 4 shows an example of the calibration efficiency in different ways. In the case of directly optimization, for the same initial condition and settings, because of the large number of parameters to be optimized and complex relationship among them, it is hard to search the optimum parameter set or even conform the convergence diagnosis. In other words, it means the convergence criteria were not satisfied within 


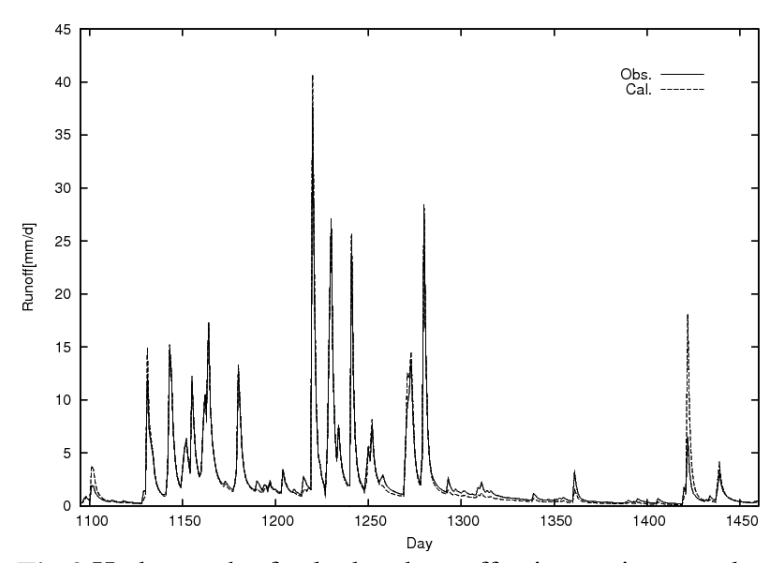

Fig.3 Hydrograph of calculated runoff using optimum values of parameters and perfect runoff, 1985.

Table 4 Efficiency of optimizing directly and multi-step optimization scheme

\begin{tabular}{cccc}
\hline & \multicolumn{2}{c}{ Number of iteration } & \\
\cline { 2 - 3 } & Max. & Stopping & \\
\hline Optimize directly & 500000 & 500000 & No \\
Multi-step opt.: & & & \\
Step 1 & 500000 & 17745 & Yes \\
Step 2 & 500000 & 63855 & Yes \\
Step 3 & 500000 & 94530 & Yes \\
\hline
\end{tabular}

the maximum number of iterations. In this condition, optimization process becomes time-consuming and depends on the selection of initial values strongly.

From the multi-step optimization results, it could be seen that most of parameters were converge to the true values quickly with small errors. Which should be noted is the differences between the true values and optimum values of the parameters in the first step (Cp, Cep) and the parameters that controlling runoff generation (WM, WUM, WLM). The sensitivity analysis by $\mathrm{Lu}$ and $\mathrm{Li}$ (2010) showed that $\mathrm{Cp}$ and Cep had strong interaction with each other. The effect caused by increase of $\mathrm{Cp}$ will be partly canceled by increasing Cep. In addition, the initial condition of study basin had effects on parameter optimization. It affects the runoff generation process so that leading to inaccurate estimation for parameters. As only $\mathrm{Cp}$ and Cep is optimized in the first step and other parameters are kept to be constants, the inaccurate initial condition may lead to the optimized $\mathrm{Cp}$ and Cep deviate the true values. Then this error was kept and affected the following optimization steps. This effect could be reduced by setting the initial condition and soil water state close to the actual condition. For example, we could select the hydrological records which are similar with our case, and use the average values of these records as the initial condition setting.
Table 5 Optimum values of parameters in the Xinanjiang model for observed data.

\begin{tabular}{ccccc}
\hline \multirow{2}{*}{ Para. } & \multicolumn{4}{c}{ Parameter values } \\
\cline { 2 - 5 } Group 1: & Start from & Step 1 & Step 2 & Step 3 \\
Cp & & 0.9565 & 0.9565 & 0.9565 \\
Cep & & 0.8349 & 0.8349 & 0.8349 \\
Group 2: & & & & \\
SM & 15.0 & 15.0 & 29.96 & 29.96 \\
EX & 1.5 & 1.5 & 1.0156 & 1.0156 \\
KI & 0.3 & 0.3 & 0.5824 & 0.5824 \\
KG & 0.4 & 0.4 & 0.1072 & 0.1072 \\
cs & 0.35 & 0.35 & 0.5958 & 0.5958 \\
ci & 0.85 & 0.85 & 0.5971 & 0.5971 \\
cg & 0.9 & 0.9 & 0.9813 & 0.9813 \\
Group 3: & & & & \\
b & 0.2 & 0.2 & 0.2 & 0.0562 \\
imp & 0.01 & 0.01 & 0.01 & 0.0361 \\
WM & 160.0 & 160.0 & 160.0 & 173.88 \\
WUM & 20.0 & 20.0 & 20.0 & 21.64 \\
WLM & 60.0 & 60.0 & 60.0 & 51.38 \\
C & 0.1 & 0.1 & 0.1 & 0.0406 \\
Annual & & & & \\
NASH & & 0.9862 & 0.9874 & 0.9886 \\
Daily & & & & \\
NASH & & 0.5867 & 0.7370 & 0.7284 \\
\hline & & & &
\end{tabular}



Fig.4 Hydrograph of Calculated Runoff Using Optimum Values of Parameters and Observed Runoff, 1985.

\section{(2) Actual practice}

Then the same series of observed runoff data in Misai Basin were used instead of perfect data to check the capacity of this scheme in actual events. As the data of soil condition is absent, we used an average record of soil water at each beginning day of 6 years (1993-1998) as the initial condition. The optimization process started from a set of parameter values which is commonly used in China. The optimum parameters and calibration result could be seen in Table 5 and Fig. 4. 
It could be seen that, although the optimum values of $\mathrm{KI}$ and $\mathrm{KG}$ are reasonable, they are not consistent with the empirical values. The $\mathrm{KI}$ is larger while $\mathrm{KG}$ is smaller than their empirical values. It is possible to conclude that the multi-step optimization scheme is capable of searching an optimum parameter set in the feasible parameter space, but it still remains difficulties on parameter identifiability to find the 'best' parameter set. Moreover, the value of SM usually tended to the maximum of feasible range. Because the SM represents the free water capacity which is sensitive for runoff components division, that may be the reason for the unusual optimum values of parameters that controlling runoff separation and runoff concentration. Generally, repeating the whole optimization process and for each repetition use the optimum values got from last repetition as the initial values could improve the optimization result, but this improvement is not so significant. Maybe more comprehensive objective function should be considered to improve this optimization step.

\section{CONCLUSION}

In this paper, a multi-step optimization scheme is developed for calibrating parameters in the Xinanjiang model. Accounting for the time scale dependency of parameters, the total 15 parameters in the Xinanjiang model were divided into three groups and optimized group by group. Some case studies were designed to test the efficiency of the scheme.

Compared with optimizing all the parameters directly without dividing into groups, the multi-step optimization scheme could enhance the efficiency of parameter calibration in the Xinanjiang model. The tests show this scheme under ideal condition could converge to the true values of parameters to be optimized. For the actual practice, the multi-step optimization scheme is capable of searching the optimum parameter set for Xinanjiang model, and the simulation results are acceptable.
1) Zhao, R. J., Liu, X. R., and Singh, V. P. : The Xinanjiang model, Computer models of watershed hydrology, Water Resources Publications, pp.215-232, 1995.

2) Gan, T. Y., and Biftu, G. F. : Automatic calibration of conceptual rainfall-runoff models: Optimization algorithms, catchment conditions and model structure. Water Resources Research, vol.32, pp.3513-3524, 1996.

3) Rosenbrock, H. H. : An automatic method for finding the greatest or least value of a function, The computer Journal, Vol.3(3), pp.175, 1960.

4) Duan, Q. Y., Gupta, V. K., and Sorooshian, S., Communicated by L. C. W. Dixon : Shuffled Complex Evolution Approach for effective and efficient global minimization, Journal of Optimization Theory Applications, Vol.76(3), pp.501-521, 1993.

5) Yapo, P. O., Gupta, H. V. and Sorooshian, S. : Multi-objective global optimization for hydrologic models, Journal of Hydrology, Vol.204(1-4), pp.83-97, 1998.

6) Beven, K. and Binley, A. : Future of distributed models: Model calibration and uncertainty prediction. Hydrological processes, Vol.6(3), 279-298, 1992.

7) Beven, K. : Prophecy, reality and uncertainty in distributed hydrological modeling, Advances in Water Resources, Vol.16(1), pp.41-51, 1993.

8) Chen, J. F., Zhang, W. C. and Wu, B. : Multi-objective calibration with predictive uncertainty analysis for conceptual hydrological models, Bulletin of Soil and Water Conservation, Vol.28(3), pp.107-112, 2008.

9) Hapusrachchi, H. A. P., Li Z. J., and Wang S. H. : Application of SCE-UA method for calibrating the Xinanjiang Watershed Model, Journal of Lake Science, Vol.12(4), pp.304-314, 2001.

10) Zhang H. G., Wang J. X., Liu P., Peng D. Z. and He X. L. : Comparative Study of Parameter Optimization Algorithms for Hydrological Model, Journal of Shihezi University (Natural Science), No.3, pp.61-85, 2002.

11) Lu, M. J., and Li X. : Time Scale Dependent Sensitivity of Xinanjiang model Parameters, submitted to IWRM5, 2010.

12) Vrugt, J. A., Gupta, H. V., Bouten, W., and Sorooshian, S. : A Shuffled Complex Evolution Metropolis algorithm for estimating posterior distribution of watershed model parameters, Calibration of Watershed Models, American Geophysical Union, pp.105-112, 2002.

13) Vrugt, J. A., Gupta, H. V., Bouten, W., and Sorooshian, S. : A Shuffled Complex Evolution Metropolis algorithm for optimization and uncertainty assessment of hydrologic model parameters, Water Resources Research, Vol.39(8), 1201, 2003.

14) Nash, J. E., and Sutcliffe, J. V. : River flow forecasting through conceptual models part 1- A discussion of principles, Journal of hydrology, Vol.10(3), pp.282-290, 1970.

\section{REFERENCES}

(Received September 30, 2011) 